\title{
Prevalence of Genes Encoding Outer Membrane Virulence Factors Among Fecal Escherichia coli Isolates
}

\author{
Ahmad Rashki ${ }^{{ }^{*}}$, Hussein Ali Abdi ${ }^{2}$, Milad Shookohi ${ }^{3}$ \\ ${ }^{1}$ Associate Professor in Molecular Genetics and Microbiology, Department of Pathophysiology, Faculty of Vet-Medicine, \\ University of Zabol, Zabol, Iran \\ ${ }^{2} \mathrm{PhD}$ Student in Molecular Genetics, Faculty of Sciences, University of Sistan and Baluchestan, Zahedan, Iran \\ ${ }^{3} \mathrm{MSc}$ in Molecular Genetics, Department of Biology, Faculty of Basic Sciences, University of Zabol, Zabol, Iran
}

\author{
*Correspondence to \\ Ahmad Rashki; Department of \\ Pathophysiology, Faculty of Vet- \\ Medicine, University of Zabol, \\ Zabol, Iran. \\ Tel: +98-5424822250; \\ Fax: +98-5424822251; \\ Email: ah_rashki@usal.es
}

Received October 26, 2016 Accepted March 6, 2017

Published online March 18, 2017

\begin{abstract}
Objective: Escherichia coli is commensal bacterium of human intestine. The gut is a common pool of $E$. coli isolates causing urinary tract infections (UTIs). Some of fecal E. coli (FeEC) by the possession of certain virulence factors is able to cause diseases in human and other mammalian models. To evaluate the health threats coordinated with a given fecal source of $E$. coli strains, we determined the frequency of genes expressing virulence determinants in fecal $E$. coli isolates collected from human feces in Zabol, southeast of Iran.

Methods: Escherichia coli isolates $(n=94)$ were separated from the feces of patients attending teaching hospitals, and screened for various virulence genes: fimH, his, hlyA, ompT, irp2, iucD, iroN, and cnf1 by using the multiplex polymerase chain reaction (PCR) method.

Results: The prevalence of virulence genes was as follows: adhesins (fimH, 98\% and iha, 26\%), alpha-hemolysins ( $h l y A, 10 \%)$, outer membrane protease (ompT, 67\%), aerobactin (iucD, 67\%), iron-repressible protein (irp2, 91\%) and salmochelin (iroN, 33\%) and cytotoxic necrotizing factor 1 (cnf1). According to the diversity of different virulence genes, the examined isolates exhibited 29 different patterns.

Conclusion: Our results demonstrated that most of the assessed isolates harbored several virulence factors. Our findings propose possibility of human feces serving as a source for pathogenic organisms, supporting the notion that fecal materials of humans play a role in the epidemiological chain of extra-intestinal pathogenic E. coli. This is the first report of the frequency of virulence factors among $E$. coli isolates collected from human feces in Iran. Keywords: Fecal Escherichia coli, Major virulence factors, Multiplex PCR
\end{abstract}

and are frequently associated with human diseases.

According to multiple virulence determinants, E. coli bacteria can be categorized into 3 major subclasses: commensal, intestinal pathogenic and extra intestinal pathogenic E. coli (ExPEC). ${ }^{6}$ It has been shown that intestinal and ExPEC strains may be extended from commensal types through obtaining of virulence mediators. ${ }^{7}$ ExPEC exhibit significant genetic heterogenicity and represents a wide spectrum of virulence-related factors such as adhesins, toxins, and iron up taking molecules, lipopolysaccharides, polysaccharide capsules, and invasions, which can be expressed on mobile genetic segments including plasmids, bacteriophages, and pathogenici-

Copyright (C) 2017 The Author(s); Published by Zabol University of Medical Sciences. This is an open-access article distributed under the terms of the Creative Commons Attribution License (http://creativecommons.org/licenses/by/4.0), which permits unrestricted use, distribution, and reproduction in any medium, provided the original work is properly cited. 
ty-associated islands (PAIs). ${ }^{8}$ The fecal flora from the hosts provides the most common source for infecting $E$. coli strain. ${ }^{9}$ The UTI-causing strains that are commonly called UPEC are obtained by faces, the place of their entrance to the urinary tract via colonization of the vaginal introitus and the periurethral location. ${ }^{10}$ E. coli strains residing within intestinal tract and promoting UTI typically contain virulence genes necessitated for colonization of the urinary tract. On the other hand, it seems that some of FeEC strains carrying many genes that encode virulence factors and can cause serious disease at diverse extraintestinal sites.

Multiple studies have been done in different parts of Iran in order to investigate the frequency of virulence genes among UPEC. ${ }^{11-13}$ However, there is no information about the frequency of virulence mediators in FeEC isolated from human in Iran. Therefore, this study was conducted to examine the distribution of 8 virulence factors, including type 1 fimbriae ( $\mathrm{fim} H$ ), iron-regulated gene homologue adhesion (iha), alpha-hemolysin ( $h l y A)$, outer membrane protease $(o m p T)$, aerobactin $(i u c D)$, yersiniabactin (irp2), salmochelin receptor (iroN) and cytotoxic necrotizing factor 1 ( $c n f 1)$ in E. coli strains isolated from human feces in Zabol, southeast of Iran, by using the multiplex polymerase chain reaction (PCR) method.

\section{Methods}

Bacterial Isolates

The sample size was calculated as described by Charan and Biswas. ${ }^{14}$ Swabs were collected directly from stool samples of the patients with diarrhea admitted to teaching hospitals in Zabol, Iran during July 2014 through October 2014. Samples were suspended into Cary-Blair transport media (Laboratorios Conda, S.A., Spain) and transported to laboratory on ice where one loop from each sample was streaked directly on MacConkey agar (HiMedia Laboratories) within 4 hours after collection. Plates were incu- bated at $37^{\circ} \mathrm{C}$ for $18-24$ hours, and up to 3 colonies with typical appearance of $E$. coli were selected and subjected to biochemical tests including oxidase, indole, methyl red, Voges-Proskauer, nitrate reduction, urease production, Simmons' citrate agar (HiMedia Laboratories), and various sugar fermentation. ${ }^{15,16}$

\section{Extraction of DNA}

The bacteria were isolated from $1 \mathrm{~mL}$ of the E. coli culture grown for 18 hours at $37^{\circ} \mathrm{C}$. The bacterial DNA was extracted by boiling methods. ${ }^{17}$ Briefly, all E. coli isolates were cultivated overnight (16 hours) in $5 \mathrm{~mL}$ Luria-Bertani (LB) broth (HiMedia Laboratories) in a shaking incubator $(200 \mathrm{rpm})$ at $37^{\circ} \mathrm{C}$. Two milliliters of bacterial isolates were then pelleted, suspended in $200 \mu \mathrm{L}$ of sterile double-distilled water and boiled at $95^{\circ} \mathrm{C}$ for 10 minutes. The mixture was cooled on ice (5 minutes), and the supernatant was collected following centrifugation (13000 rpm 5 minutes). After centrifugation, the supernatants were kept as DNA at $-20^{\circ} \mathrm{C}$ until applied for PCR.

Detection of Putative Virulence Genes Using the Multiplex PCR Method

Prevalence of putative virulence genes in FeEC isolates was determined by multiplex PCR. ${ }^{3}$ Details of primer sequences, target genes and products size are shown in Table 1. Amplification of selected genes was performed by setting a net volume of $25 \mu \mathrm{L}$. The reaction contained 2 $\mu \mathrm{L}$ of DNA, $12.5 \mu \mathrm{L}$ of Taq DNA Polymerase Master Mix Red (amplicon, A/S, Denmark), $1 \mu \mathrm{L}$ of primers (30 pmol concentration for each) (Pishgam, Iran) and $8.530 \mathrm{pmol}$ $\mathrm{ddH}_{2} \mathrm{O}$. The multiplex PCR was performed considering an initial phase of denaturation $\left(94^{\circ} \mathrm{C}, 5\right.$ minutes), followed by 35 cycles consisting of denaturation $\left(94^{\circ} \mathrm{C}\right.$ for 30 seconds), annealing $\left(59^{\circ} \mathrm{C}\right.$ for 50 seconds) and extension $\left(72^{\circ} \mathrm{C}\right.$ for 70 seconds), and followed by a final extension step at $72^{\circ} \mathrm{C}$ for 5 minutes. Amplification was performed

Table 1. Primers for the Multiplex PCR Assays

\begin{tabular}{|c|c|c|c|c|}
\hline Virulence Factor & Target gene & Primer & Primer Sequences (5'-3') & Size of Product(bp) \\
\hline \multirow[t]{2}{*}{ Cytotoxic necrotizing factor 1} & cnf1 & cnf1-F & AGGCAGGAATAAACCAGGAGGT & 1286 \\
\hline & & cnf1-R & ACGAGCAGAATTTGACACACGA & \\
\hline \multirow[t]{2}{*}{ Outer membrane protease } & ompT & ompT-F & TGCGATCAGCTCTTTTGCTTCT & 144 \\
\hline & & ompT-R & AGTTGACTGACTTTTCGGCCTC & \\
\hline \multirow[t]{2}{*}{ Yersiniabactin } & irp2 & $\operatorname{irp} 2-\mathrm{F}$ & AGCATCGCCTGCTAAAACTGAA & 623 \\
\hline & & irp2-R & CAGACGATGCAGGGCGTTATTA & \\
\hline \multirow[t]{2}{*}{ Iron-regulated gene homologue adhesion } & iha & iha-F & CTGGAAGTCAGCATTCGTGGAA & 934 \\
\hline & & iha-R & GATGCСАСТCATCCTCAGCAAA & \\
\hline \multirow[t]{2}{*}{ Alpha-hemolysin } & hlyA & hlyA-F & GTTAGCGGGTGTCACCAGAAAT & 1361 \\
\hline & & hlyA-R & GTGTGATTACCCTGCCGTCTTT & \\
\hline \multirow[t]{2}{*}{ Salmochelin receptor } & $\operatorname{iroN}$ & iroN-F & CGGTTCCTGGCACGAATATCAT & 1048 \\
\hline & & iroN-R & TTTTGGGATTTCCCCAACCTGG & \\
\hline \multirow[t]{2}{*}{ Aerobactin } & iuc $D$ & iucD-F & ATGGCATCACTGCCGATTCTTT & 534 \\
\hline & & iucD-R & AGTGAGTTAAAGCAGCAGCСТC & \\
\hline \multirow[t]{2}{*}{ Type 1 fimbriae } & $\mathrm{fimH}$ & fimH-F & ATTCCTCACAATCAGCGCACTT & 170 \\
\hline & & fimH-R & ATCAGCAGTACAGCAAACAGGG & \\
\hline
\end{tabular}


using a gradient Eppendorf's Mastercycler ${ }^{\circledR}$ Pro (Eppendorf, Germany). The multiplex PCR products were separated by agarose gel $2 \%$ electrophoresis and visualized under UV-induced fluorescence. A 100 bp DNA ladder (Fermentase) was used as size standard (Figure 1). Amplification identities were confirmed by restriction analysis.

\section{Restriction Analysis}

Amplified fragments of selected genes were confirmed by restriction analysis. Restriction patterns of 8 sequences were obtained with the Webcutter 2.0, online software (http://rna.lundberg.gu.se/cutter2/). The hlyA, iha, irp2 and iroN gene sequences were restricted with $T f i$ I endonuclease followed ompT, iucD and fim H sequences restricted with $A l u I$ endonuclease. MspI endonuclease was chosen for restriction of $c n f 1$ sequence. Restriction conditions were identical in all cases. Each $30 \mu \mathrm{L}$ reaction mixture contained $1 \mu \mathrm{L}$ of restriction endonuclease, $8 \mu \mathrm{L}$ of PCR product, $3 \mu \mathrm{L}$ of specific endonuclease enzyme buffer and $18 \mu \mathrm{L}$ of sterilized $\mathrm{dH}_{2} \mathrm{O}$. After overnight incubation, the restriction products were determined by electrophoresis of the digested DNA in 2\% agarose gel.

\section{Results}

The frequency of virulence genes collected from $E$. coli isolates of fecal samples is shown in Figure 2. In total, 94/94 (100\%) of FeEC isolates represented with at least one of the studied virulence genes, of which 1 (1\%), 18 (19\%), 10 (11\%), and 29 (31\%) were detected harboring $1,2,3$, and 4 virulence factors, respectively. Twenty-nine different virulence combinations were found among fecal E. coli isolates (Table 2). FeEC11 pattern was determined by the presence of the irp2, ompT, iucD and fimH genes which presented the most identified pattern, detected in $19(20 \%)$ isolates. $\mathrm{fimH}$ was the frequent virulence gene identified in all 92(98\%) FeEC isolates. Among the iron-acquisition genes, irp 2 was the most prevalent gene and was identified in $86(91 \%)$ isolates, while $i u c D$ and iroN genes were detected in 63 (67\%) and 31 (33\%) isolates respectively. The secretory virulence genes including hly and cnf were present in $9(10 \%)$ and $3(3 \%)$ FeEC isolates, respectively. The ompT gene was identified in $63(67 \%)$ and the iha was detected in $24(26 \%)$ isolates. The association of 7 genes was recognized in FeEC1 and FeEC2 patterns (3 isolates). One isolate harbored fimH only (FeEC29). The 2 FeEC3 and FeEC4 patterns included isolates possessing a combination of 6 amplified genes (Table 2).

The detected virulence genes were confirmed by restriction analysis: iha, iroN, irp2 and hlyA amplicons (934, 1084, 623 and 1361 bp respectively) were restricted with TfiI endonuclease enzyme and yielded fragments of 465, 267, 129 and 73 bp; 1004 and 80 bp; 426 and 197 bp; and 1030 and $331 \mathrm{bp}$ respectively. The $c n f 1$ amplicon (1286 bp) was restricted with $M s p I$ and yielded fragments of 467, 404, 261 and $154 \mathrm{bp}$. The detected virulence genes were confirmed by restriction analysis: iucD, ompT and fimH amplicons (534, 144 and 170 bp respectively) and were

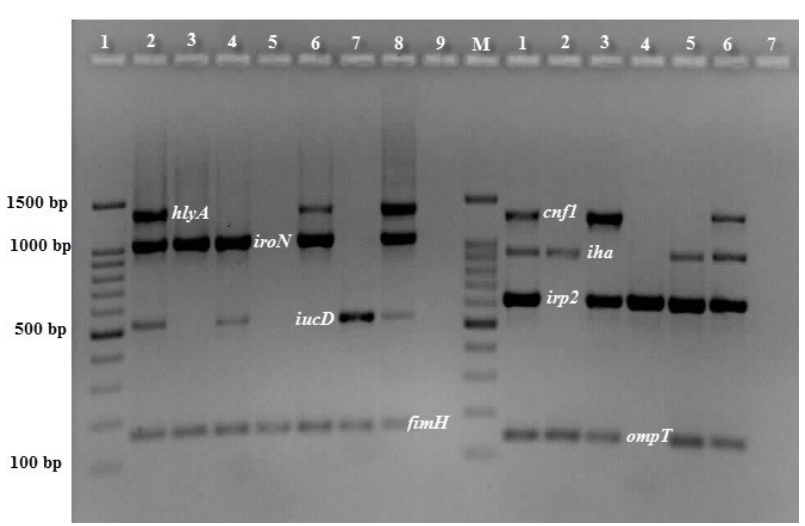

Figure 1. Electrophoresis of Virulence Selected Genes Among FeEC Isolate Obtained by Multiplex PCR.

Abbreviations: FeEC, fecal Escherichia coli; PCR, polymerase chain reaction.

Each band is indicated by the names of the virulence genes. Lines 1 and " $\mathrm{M}$ " denote 100 bp DNA marker.

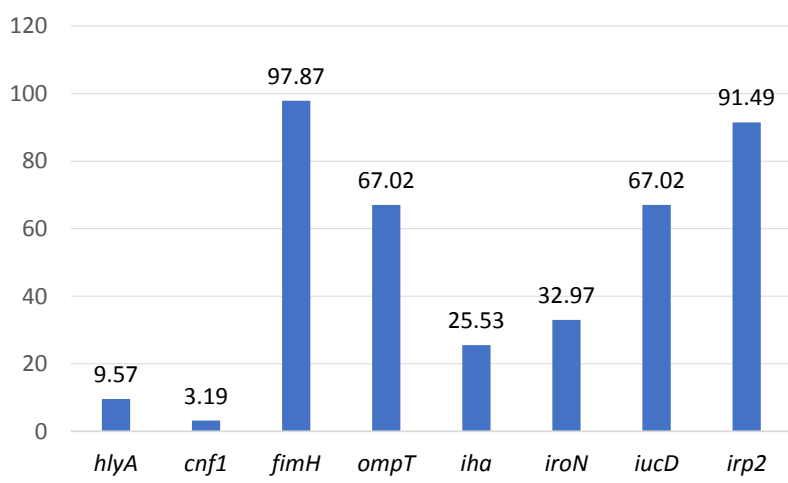

Figure 2. Prevalence of Virulence Genes Among 94 FeEC Isolates Collected From Human Feces.

Abbreviation: FeEC, fecal Escherichia coli.

restricted with AluI endonuclease enzyme and yielded fragments of 386 and 148 bp; 135 and 9 bp; and 109 and 61 bp respectively (Figure 3 ).

\section{Discussion}

The lower gastrointestinal tract is considered as the richest pool of UTI generating organisms. Some strains can colonize the vagina and urinary tract. ${ }^{18}$ The variety and heterogeneity of virulence factors, including adhesins, toxins and siderophores emerge to be momentous for E. coli strains and make the development of multiplex PCR method especially significant. In this work, to our knowledge for the first time in Iran, we assessed the prevalence of virulence gene profile in 94 FeEC isolates Understanding the distribution of virulence determinants in FeEC isolates is important to evaluate their relative contribution to the extraintestinal infection. We also assessed multiplex PCR assays to detect virulence factors utilizing a combination of primers previously reported. ${ }^{3}$

Our findings showed that FimH adhesion was the most prevalent virulence factor detected. This factor occurred in 92 (98\%) FeEC isolates as seen in Table 2. Similar results were obtained by previous studies, ${ }^{18,19}$ whereas the 
Table 2. Virulence Gene Patterns Identified Among the Studied Isolates

\begin{tabular}{|c|c|c|c|c|c|c|c|c|c|}
\hline \multirow{2}{*}{ Patterns } & \multicolumn{8}{|c|}{ Urovirulence Genes } & \multirow{2}{*}{ No. of Strains } \\
\hline & cnf1 & iha & irp2 & ompT & hlyA & iroN & iucD & $\mathrm{fimH}$ & \\
\hline FeEC1 & - & + & + & + & + & + & + & + & 2 \\
\hline FeEC2 & + & - & + & + & + & + & + & + & 1 \\
\hline FeEC3 & - & - & + & + & + & + & + & + & 2 \\
\hline FeEC4 & - & + & + & + & - & + & + & + & 3 \\
\hline FeEC5 & - & + & + & - & + & + & - & + & 1 \\
\hline FeEC6 & - & - & + & + & - & + & + & + & 12 \\
\hline FeEC7 & - & - & + & - & + & + & - & + & 1 \\
\hline FeEC8 & - & + & + & - & - & + & + & + & 1 \\
\hline FeEC9 & - & - & + & + & - & - & - & + & 4 \\
\hline FeEC10 & - & + & + & - & - & - & + & + & 6 \\
\hline FeEC11 & - & - & + & + & - & - & + & + & 19 \\
\hline FeEC12 & - & + & + & + & - & - & - & + & 1 \\
\hline FeEC13 & - & - & + & + & - & + & - & - & 1 \\
\hline FeEC14 & - & + & + & - & - & - & - & + & 1 \\
\hline FeEC15 & + & - & + & + & - & + & - & + & 1 \\
\hline FeEC16 & + & - & + & + & - & - & + & + & 1 \\
\hline FeEC17 & - & + & - & + & - & + & + & + & 1 \\
\hline FeEC18 & - & - & + & + & + & + & - & + & 1 \\
\hline FeEC19 & - & - & + & + & - & + & - & + & 2 \\
\hline FeEC20 & - & - & + & - & + & - & + & + & 1 \\
\hline FeEC21 & - & + & + & + & - & - & + & + & 8 \\
\hline FeEC22 & - & - & + & - & - & + & - & + & 1 \\
\hline FeEC23 & - & - & + & - & - & - & + & + & 4 \\
\hline FeEC24 & - & - & + & - & - & - & - & + & 11 \\
\hline FeEC25 & - & - & + & - & - & - & + & - & 1 \\
\hline FeEC26 & - & - & - & - & - & + & - & + & 1 \\
\hline FeEC27 & - & - & - & - & - & - & + & + & 1 \\
\hline FeEC28 & - & - & - & + & - & - & - & + & 4 \\
\hline FeEC29 & - & - & - & - & - & - & - & + & 1 \\
\hline Total & 3 & 24 & 86 & 63 & 9 & 31 & 63 & 92 & 94 \\
\hline
\end{tabular}

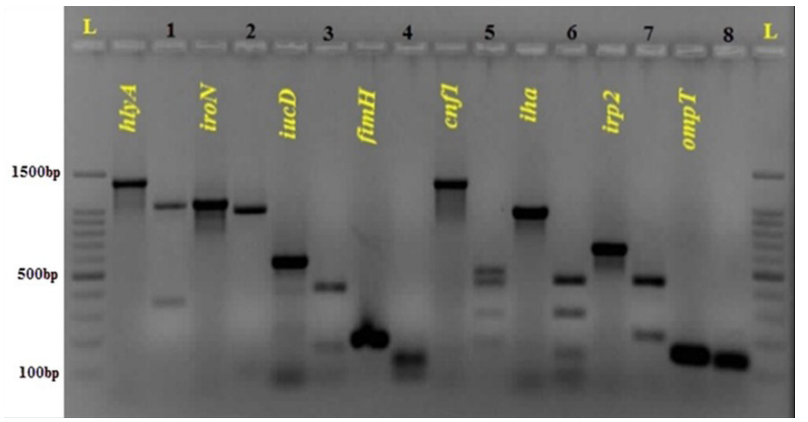

Figure 3. Gel Electrophoresis for Digestion Results of Virulence Genes in FeEC Isolates.

L: Lader; Lane 1. Digest of hlyA (1361bp) with Tfil enzyme and production of fragments $331 \mathrm{bp}$ and $1030 \mathrm{bp}$; Lane 2. Digest of iroN (1084 bp) with Tfil enzyme and production of fragments 80 bp and 1004 bp; Lane 3. Digest of iucD (534 bp) with Alul enzyme and production of fragments $148 \mathrm{bp}$ and $386 \mathrm{bp}$; Lane 4 . Digest of fimH (170 bp) with Alul enzyme and production of fragments 61 bp and 109 bp; Lane 5. Digest of cnf1 (1286 bp) with Mspl enzyme and production of fragments $154 \mathrm{bp}, 261 \mathrm{bp}, 404 \mathrm{bp}$ and $467 \mathrm{bp}$; Lane 6. Digest of iha (934 bp) with Tfil enzyme and production of fragments 73 bp, 129 bp, 267 bp and 465 bp; Lane 7. Digest of irp2 (623 bp) with Tfil enzyme and production of fragments $197 \mathrm{bp}$ and 426 bp; Lane 8. Digest of ompT (144 bp) with Alul enzyme and production of fragments $9 \mathrm{bp}$ and $135 \mathrm{bp}$ frequency of the iha gene, as other adhesion, was $26 \%$ (24/94). Our laboratory previously showed that the prevalence of iha gene was $29 \%$ among UPEC isolates. ${ }^{20}$ However, no significant differences were observed in relation to iha gene. According to our knowledge, to date, no reports have been published about the prevalence of iha gene among FeEC isolates. The findings of our study indicated that the presence of the $h l y A$ and $c n f 1$ genes was $10 \%$ and $3 \%$ respectively.

In another study, Usein et $\mathrm{al}^{21}$ demonstrated that the $h l y A$ and $c n f 1$ genes were found in $35 \%$ of E. coli bacteria recovered from the fecal flora of healthy adult humans. The results showed the variation geographical distribution of these genes. The prevalence of the $c n f 1$ gene was different in fecal isolates studied by other investigators. ${ }^{22,23}$ In accordance with our study, Obiet et $\mathrm{al}^{24}$ also described that the $c n f 1$ gene was expressed in $3.5 \%$ of $E$. coli isolates collected from diarrheic stool samples in South Africa. In another study, our group demonstrated that $c n f 1$ gene was more frequently detected in UPEC (28\%) in comparison with FeEC isolates (3\%). ${ }^{20}$ Particularly, ExPEC expresses a richness of apparently excessive iron obtaining systems, including the salmochelin, yersiniabactin, and aerobactin 
siderophores. In accordance, we observed in our study a very high prevalence of the iron acquisition genes; irp2 (91\%), iucD (67\%) and iroN (33\%). The irp gene cluster is mapped within the high pathogenicity island (HPI) described primarily in Yersinia spp. and horizontal gene transfer has caused it to be present in intestinal and extra intestinal clinical E. coli strains. ${ }^{25}$ The iroN gene, that is situated on the iro $A$ gene cluster encodes a receptor which is responsible for iron uptake mediated by the siderophores salmochelins, contributes to the virulence of UPEC. This interaction facilitates transport of the complex into the bacterial cytosol. ${ }^{26}$ The virulence genes of UPEC bacteria such as iroN, iucD and irp2 have been previously recorded from Iran. ${ }^{11,12}$ However, according to published data, there is no information on the occurrence of these genes in FeEC isolates.

The distribution of the ompT gene among the studied isolates was also similar to that in the previously reported data. ${ }^{27}$ OmpT rather seems as a conserved protease executing in metabolism of $E$. coli derived from secretory proteins. This outer membrane protease contributes to the destruction of several proteins interacting with the outer membrane. ${ }^{28}$ However, there is no report about the prevalence of ompT gene among FeEC isolates in Iran. The analysis of the association between the presences of different combinations of virulence genes among FeEC isolates, allowed us to divide the tested isolates into 29 virulence patterns noted FeEC1 to 29. Our results revealed the complexity of the properties of virulence markers in fecal E. coli isolates. The pattern FeEC6 included strains simultaneously positive for $i r p 2^{+}, o m p T^{+}, i u c D^{+}, i r o N^{+}$and $\mathrm{fimH}^{+}$(12 isolates). The prevalence of genes coding for the 2 adhesion pathways (type 1 fimbriae and iha) which confers the ability to colonization among 24 isolates were fit with those published by other investigators. ${ }^{3,29}$ Among FeEC isolates, 89 isolates contain genes encoding an iron acquisition protein (yersiniabactin, aerobactin or salmochelin receptor). The maximum number of detected amplicons in 1 isolate was 6 . The main novel finding is the high occurrence of genes of outer membrane virulence proteins among FeEC isolates in Zabol, southeast of Iran. Apart from the high occurrence of virulence genes, various virulence determinants were also revealed in fecal $E$. coli strains. In our analysis, 74\% (70/94) of the bacteria were represented with various virulence genes (4 or more genes). It is comprehensible that the recurrence and prevalence of virulence features of fecal E. coli strains are different in other regions of Iran. Probably, geographical differences, cultural habitants, dietary features, public and hospital health policies, weather climate of each region and even sampling methods may exert high impacts on detection rate of virulence factors of FeEC isolates. Nonetheless, further research on the expression of virulence genes and molecular typing methods covering wider geographical areas in Iran is needed to determine the distribution pattern of virulence determinants and develop effective strategies to treat FeEC -induced diseases. This may constitute a limitation of our study.

\section{Conclusion}

The FeEC isolates expressing virulence genes may be regarded as an important organism for extra-intestine infections in Iran. The current work is the first report which identified virulence genes among FeEC isolates in Iran. The multiplex PCR designed in the present study successfully screened FeEC isolates for various E. coli-related virulence genes. Further studies in other parts of Iran are needed to identify virulent factors and to ascertain the pathophysiology of such infectious agents to consider possible prevention interventions.

\section{Ethical Approval}

We obtained informed consent form our participants.

\section{Competing Interests}

Authors declare that they have no conflict of interest.

\section{Acknowledgments}

We wish to thank the staff of the laboratory of microbiology of the faculty of vet-medicine at university of Zabol.

\section{References}

1. Gruneberg RN. Relationship of infecting urinary organism to the faecal flora in patients with symptomatic urinary infection. Lancet. 1969;2(7624):766-768.

2. Johnson JR, Kaster N, Kuskowski MA, Ling GV. Identification of urovirulence traits in Escherichia coli by comparison of urinary and rectal $E$. coli isolates from dogs with urinary tract infection. J Clin Microbiol. 2003;41(1):337-345.

3. Rashki A. Cervico-vaginopathogenic Escherichia coli in Iran: serogroup distributions, virulence factors and antimicrobial resistance properties. Microb Pathog. 2014;75:29-34 doi:10.1016/j.micpath.2014.1008.1004.

4. Dobrindt U, Janke B, Piechaczek K, et al. Toxin genes on pathogenicity islands: impact for microbial evolution. Int J Med Microbiol. 2000;290(4-5):307-311. doi:310.1016/ S1438-4221(1000)80028-80024.

5. Stecher B, Hardt WD. The role of microbiota in infectious disease. Trends Microbiol. 2008;16(3):107-114. doi:110.1016/j.tim.2007.1012.1008.

6. Finlay BB, Falkow S. Common themes in microbial pathogenicity revisited. Microbiol Mol Biol Rev. 1997;61(2):136-169.

7. Russo TA, Johnson JR. Proposal for a new inclusive designation for extraintestinal pathogenic isolates of Escherichia coli: ExPEC. J Infect Dis. 2000;181(5):17531754. doi: $1710.1086 / 315418$.

8. Rodriguez-Siek KE, Giddings CW, Doetkott C, et al. Comparison of Escherichia coli isolates implicated in human urinary tract infection and avian colibacillosis. Microbiology. 2005;151(Pt 6):2097-2110. doi:2010.1099/ mic.2090.27499-27490.

9. Clermont O, Bonacorsi S, Bingen E. Rapid and simple determination of the Escherichia coli phylogenetic group. Appl Environ Microbiol. 2000;66(10):4555-4558

10. Wilson BA, Salyers AA, Whitt DD, Winkler ME. Bacterial pathogenesis: a molecular approach: American Society for Microbiology (ASM); 2011.

11. Momtaz H, Karimian A, Madani M, et al. Uropathogenic 
Escherichia coli in Iran: serogroup distributions, virulence factors and antimicrobial resistance properties. Ann Clin Microbiol Antimicrob. 2013;12:8. doi:10.1186/1476-07111112-1188.

12. Abdi HA, Rashki A. Comparison of virulence factors distribution in uropathogenic E. coli Isolates from phylogenetic groups B2 and D. Int J Enteric Pathog. 2014;2(4):e21725.

13. Charan J, Biswas T. How to calculate sample size for different study designs in medical research? Indian J Psychol Med. 2013;35(2):121-126. doi:110.4103/0253-7176.116232.

14. Maluta RP, Stella AE, Riccardi K, et al. Phenotypical characterization and adhesin identification in Escherichia coli strains isolated from dogs with urinary tract infections. Braz J Microbiol. 2012;43(1):375-381. doi:10.1590/S1517838220120001000045.

15. Chen YM, Wright PJ, Lee CS, Browning GF. Uropathogenic virulence factors in isolates of Escherichia coli from clinical cases of canine pyometra and feces of healthy bitches. Vet Microbiol. 2003;94(1):57-69. doi:10.1016/S03781135(1003)00063-00064.

16. Rahdar M, Rashki A, Miri HR, Rashki Ghalehnoo M. Detection of pap, sfa, afa, foc, and fim adhesin-encoding operons in uropathogenic Escherichia coli isolates collected from patients with urinary tract infection. Jundishapur J Microbiol. 2015;8(8):e22647. doi:10.5812/jjm.22647

17. Rashki A. Cervico-vaginopathogenic Escherichia coli in Iran: Serogroup distributions, virulence factors and antimicrobial resistance properties. Microb Pathog. 2014;75:29-34. doi:10.1016/j.micpath.2014.1008.1004.

18. Moreno E, Andreu A, Pigrau C, et al. Relationship between Escherichia coli strains causing acute cystitis in women and the fecal E. coli population of the host. J Clin Microbiol. 2008;46(8):2529-2534. doi:2510.1128/JCM.00813-00808.

19. Kaczmarek A, Budzynska A, Gospodarek E. Prevalence of genes encoding virulence factors among Escherichia coli with $\mathrm{K} 1$ antigen and non-K1 E. coli strains. J Med Microbiol. 2012;61(Pt 10):1360-1365. doi:1310.1099/ jmm.1360.044263-044260.

20. Rashki A. Cervico-vaginopathogenic Escherichia coli in Iran: Serogroup distributions, virulence factors and antimicrobial resistance properties. Microb Pathog. 2014;75:29-34. doi: 10.1016/j.micpath.2014.08.004.
21. Moreno E, Andreu A, Pigrau C, et al. Relationship between Escherichia coli strains causing acute cystitis in women and the fecal E. coli population of the host. J Clin Microbiol. 2008;46(8):2529-2534. doi:10.1128/JCM.00813-08.

22. Abdi Ha, Rashki A. The phylogenetic study of uropathogenic Escherichia coli strains in Sistan of Iran. J Birjand Univ Med Sci. 2014;21(3):392-400.

23. Usein CR, Damian M, Tatu-Chitoiu D, et al. Comparison of genomic profiles of Escherichia coli isolates from urinary tract infections. Roum Arch Microbiol Immunol. 2003 J;62(3-4):137-154.

24. Yuri K, Nakata K, Katae H, Yamamoto S, Hasegawa A. Distribution of uropathogenic virulence factors among Escherichia coli strains isolated from dogs and cats. J Vet Med Sci. 1998;60(3):287-290.

25. Caprioli A, Falbo V, Ruggeri FM, et al. Cytotoxic necrotizing factor production by hemolytic strains of Escherichia coli causing extraintestinal infections. J Clin Microbiol. 1987;25(1):146-149.

26. Obi CL, Green E, Bessong PO, et al. Gene encoding virulence markers among Escherichia coli isolates from diarrhoeic stool samples and river sources in rural Venda communities of South Africa. Water SA. 2004;30(1):37-42. doi:10.4314/wsa.v4330i4311.5024

27. Schubert S, Rakin A, Karch H, Carniel E, Heesemann J. Prevalence of the "high-pathogenicity island" of Yersinia species among Escherichia coli strains that are pathogenic to humans. Infect Immun. 1998;66(2):480-485.

28. Hantke K, Nicholson G, Rabsch W, Winkelmann G. Salmochelins, siderophores of Salmonella enterica and uropathogenic Escherichia coli strains, are recognized by the outer membrane receptor Iron. Proc Natl Acad Sci U S A. 2003;100(7):3677-3682. doi:3610.1073/pnas.0737682100.

29. Marrs CF, Zhang L, Tallman P, et al. Variations in 10 putative uropathogen virulence genes among urinary, faecal and peri-urethral Escherichia coli. J Med Microbiol. 2002;51(2):138-142. doi:110.1099/0022-1317-1051-10921138.

30. Wu XY, Chapman T, Trott DJ, et al. Comparative analysis of virulence genes, genetic diversity, and phylogeny of commensal and enterotoxigenic Escherichia coli isolates from weaned pigs. Appl Environ Microbiol. 2007;73(1):8391. doi:10.1128/AEM.00990-00906. 